\title{
Iron fortification adversely affects the gut microbiome, increases pathogen abundance and induces intestinal inflammation in Kenyan infants
}

\author{
Tanja Jaeggi, ${ }^{1}$ Guus A M Kortman, ${ }^{2}$ Diego Moretti, ${ }^{1}$ Christophe Chassard, ${ }^{1}$ \\ Penny Holding, ${ }^{3}$ Alexandra Dostal, ${ }^{1}$ Jos Boekhorst, ${ }^{4}$ Harro M Timmerman, ${ }^{4}$ \\ Dorine W Swinkels, ${ }^{2}$ Harold Tjalsma, ${ }^{2}$ Jane Njenga, ${ }^{5}$ Alice Mwangi, ${ }^{5}$ Jane Kvalsvig, ${ }^{6}$ \\ Christophe Lacroix, ${ }^{1}$ Michael B Zimmermann ${ }^{1}$
}

- Additional material is published online only. To view please visit the journal online (http://dx.doi.org/10.1136/ gutjnl-2014-307720)

${ }^{1}$ Department of Health Sciences and Technology, ETH Zurich, Zurich, Switzerland ${ }^{2}$ Department of Laboratory Medicine, Radboud University Medical Center, Nijmegen, The Netherlands

${ }^{3}$ International Center for Behavioural Studies, Mombasa, Kenya

${ }^{4}$ NIZO Food Research B.V.,

Ede, The Netherlands

${ }^{5}$ Department of Food

Technology and Nutrition, University of Nairobi, Nairobi, Kenya

${ }^{6}$ University of KwaZulu-Natal, Durban, South Africa

\section{Correspondence to}

Tanja Jaeggi, Institute of Food, Nutrition and Health, ETH Zentrum, Schmelzberstrasse 7 8092 Zurich, Switzerland; tanja.jaeggi@hest.ethz.ch

Received 26 May 2014 Revised 21 July 2014 Accepted 22 July 2014 Published Online First 20 August 2014

\section{SLinked}

http://dx.doi.org/10.1136/ gutjnl-2014-308147

CrossMark

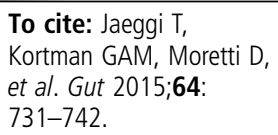

\section{ABSTRACT}

Background In-home iron fortification for infants in developing countries is recommended for control of anaemia, but low absorption typically results in $>80 \%$ of the iron passing into the colon. Iron is essential for growth and virulence of many pathogenic enterobacteria. We determined the effect of high and low dose in-home iron fortification on the infant gut microbiome and intestinal inflammation.

Methods We performed two double-blind randomised controlled trials in 6-month-old Kenyan infants ( $n=115)$ consuming home-fortified maize porridge daily for 4 months. In the first, infants received a micronutrient powder (MNP) containing $2.5 \mathrm{mg}$ iron as NaFeEDTA or the MNP without iron. In the second, they received a different MNP containing $12.5 \mathrm{mg}$ iron as ferrous fumarate or the MNP without the iron. The primary outcome was gut microbiome composition analysed by 165 pyrosequencing and targeted real-time PCR (qPCR). Secondary outcomes included faecal calprotectin (marker of intestinal inflammation) and incidence of diarrhoea. We analysed the trials separately and combined.

Results At baseline, $63 \%$ of the total microbial 165 rRNA could be assigned to Bifidobacteriaceae but there were high prevalences of pathogens, including Salmonella Clostridium difficile, Clostridium perfringens, and pathogenic Escherichia coli. Using pyrosequencing, +FeMNPs increased enterobacteria, particularly Escherichial Shigella $(p=0.048)$, the enterobacteria/bifidobacteria ratio $(p=0.020)$, and Clostridium $(p=0.030)$. Most of these effects were confirmed using $\mathrm{qPCR}$; for example, + FeMNPs increased pathogenic $E$. coli strains $(p=0.029)$. + FeMNPs also increased faecal calprotectin ( $p=0.002)$. During the trial, $27.3 \%$ of infants in $+12.5 \mathrm{mgFeMNP}$ required treatment for diarrhoea versus $8.3 \%$ in $-12.5 \mathrm{mgFeMNP}$ $(p=0.092)$. There were no study-related serious adverse events in either group.

Conclusions In this setting, provision of iron-containing MNPs to weaning infants adversely affects the gut microbiome, increasing pathogen abundance and causing intestinal inflammation.

Trial registration number NCT01111864.

\section{INTRODUCTION}

While infants have the highest rates of iron deficiency anaemia (IDA), they are also the group less well covered by universal fortification programmes. Micronutrient powders (MNP), added directly to

\section{Significance of this study}

What is already known on this subject?

- The composition of the infant gut microbiota may vary depending on dietary iron supply, but most data are from culture methods, or have come from animal experiments.

- In-home iron fortification for infants in developing countries is recommended to control anaemia, but low absorption typically results in $>80 \%$ of the iron passing into the colon.

- Two recent iron fortification trials in infants in developing countries have raised safety concerns: in Ghana, there was an increased rate of hospitalisations possibly due to diarrhoea, and in Pakistan, a small but significant increase in overall diarrhoea prevalence.

- There is little known about the composition of the African infant gut microbiota during the weaning period, or the effects of iron fortification at this age.

\section{What are the new findings?}

- This is the first controlled intervention trial to examine the effect of iron fortification on the African infant gut microbiome.

- Iron fortification modifies the gut microbiome in weaning African infants, increasing enterobacteria and decreasing bifidobacteria, and increases abundances of specific enteropathogens, for example, pathogenic Escherichia coli.

- Iron fortification in weaning African infants increases faecal calprotectin levels, indicating intestinal inflammation.

- These data provide a probable mechanism for the increases in diarrhoea seen in recent infant fortification studies.

How might it impact on clinical practice in the foreseeable future?

- Our findings suggest that, until safer formulations are available, iron fortification should not be given to all infants, but should be targeted only to infants with clear iron deficiency anaemia, while providing adequate protection from diarrhoea. 
complementary foods after cooking (in-home fortification), can be an effective approach for providing additional dietary iron and reducing anaemia rates. ${ }^{1}$ Currently, in-home fortification programmes are in place or planned in 36 countries including 10 in Sub-Saharan Africa. ${ }^{2}$

However, the safety of iron-containing MNPs (+FeMNPs) is uncertain. A 2007 WHO consultation, on the results of the Pemba trial where iron and folic acid supplementation increased child mortality, ${ }^{3}$ did not recommend the use of +FeMNPs in malaria-endemic areas because of concerns about potential increases in infection. ${ }^{4}$ Two recent fortification trials in infants with an MNP containing $12.5 \mathrm{mg}$ ferrous fumarate have raised safety concerns: in Ghana, there was an increased rate of hospitalisations possibly due to diarrhoea, ${ }^{5}$ and in Pakistan a small but significant increase in overall diarrhoea prevalence, bloody diarrhoea, and respiratory illness. ${ }^{6}$ An earlier systematic review concluded iron supplementation, but not fortification, may increase risk for diarrhoea. ${ }^{7}$ If +FeMNPs increase risk for infection and diarrhoeal disease, this would be an important adverse effect, as diarrhoea contributes to the death of $\approx 1$ in 9 under 5 -year-old children in Sub-Saharan Africa. ${ }^{8}$

The food matrix of most cereal-based and legume-based complementary foods is rich in phytic acid, a potent inhibitor of iron absorption; therefore, usually less than $20 \%$ of iron added to these foods is absorbed. ${ }^{9}{ }^{10}$ In rural African populations with high levels of inflammation and infection, absorption is likely to be even lower, as inflammation increases circulating hepcidin the major iron regulator, which reduces dietary iron absorption through binding and degradation of the iron efflux protein, ferroportin, at the basolateral membrane of the enterocytes. ${ }^{11}$ Thus, providing +FeMNPs to individuals with concurrent inflammation results in most of the iron passing unabsorbed into the colon. Once absorbed, iron in the body is bound to proteins limiting iron access to potential pathogens, and during infection, iron supply is further reduced in the extracellular compartment and remains in macrophages and enterocytes. ${ }^{12}$ There is no comparable system for sequestration of dietary iron known in the gut lumen, although neutral $\mathrm{pH}$ and the presence of defence molecules, such as lipocalin $2,{ }^{13}$ may reduce iron solubility and availability to gut microbes.

Iron is an essential, growth-limiting nutrient for many gut bacteria, competing for unabsorbed dietary iron. ${ }^{14}$ For most enteric gram-negative bacteria (eg, Salmonella, Shigella or pathogenic Escherichia coli), iron acquisition plays an essential role in virulence and colonisation. ${ }^{15}$ By contrast, lactobacilli, a major group of beneficial 'barrier' bacteria improving gut integrity and reducing colonisation by enteric pathogens, ${ }^{16}$ do not require iron, but instead rely on manganese. ${ }^{17}$ Therefore, an increase in unabsorbed dietary iron through fortification or supplementation could modify the colonic microbiota equilibrium and favour growth of pathogenic strains over 'barrier' strains.

Colonisation of the human gastrointestinal tract begins at birth and depends on the mode of delivery, hygiene and prematurity. ${ }^{18}$ The iron-binding protein, lactoferrin, in breast milk limits iron availability to the gut microbiota, ${ }^{19}$ and may have a protective effect in breastfed infants. The relatively simple gut microbiota of breastfed infants further diversifies with the introduction of complementary feeding. ${ }^{20}$ In a study comparing the gut microbiome among infants from the USA, Venezuela and Malawi, there were significant differences between sites, but a common pattern was the dominance of bifidobacteria throughout the first year of life, thereafter, bifidobacteria diminish steadily leading to the establishment of an adult-like gut microbiome at about 3 years of age. ${ }^{21}$
In a controlled trial of iron fortification in schoolchildren in Côte d'Ivoire, iron increased enterobacteria and intestinal inflammation, and decreased lactobacilli. ${ }^{22}$ In older studies in European infants using culture methods, iron induced an increase in E. coli and a decrease in bifidobacteria, ${ }^{23}$ along with higher counts of Bacteroides. ${ }^{24} \mathrm{~A}$ recent small study in US infants $(n=14)$ receiving complementary feeding regimens providing iron from fortified cereals or meat, reported an increase in Bacteroidales and decreases in bifidobacteria, Lactobacillales and Rothia. ${ }^{25}$ In infants in Pakistan, +FeMNPs caused an increase in Aeromonas species compared to the nonsupplemented control group. ${ }^{6}$

Our study aim was to determine the effects of two widely used high and low dose +FeMNPs on the gut microbiome, using barcoded 16S rRNA pyrosequencing and targeted real-time PCR (quantitative PCR, qPCR), and intestinal inflammation in Kenyan infants prone to diarrhoeal disease. Our hypotheses were that iron fortification would: (1) increase faecal enterobacteria and its ratio to bifidobacteria and/or lactobacilli; (2) favour colonisation by potential pathogens and (3) increase intestinal inflammation.

\section{MATERIALS AND METHODS}

\section{Study site and participants}

The study was conducted in Msambweni County, in southern coastal Kenya. This is a malaria-endemic area, where $40 \%$ of the paediatric hospital admissions in 2006 were due to plasmodial infections. ${ }^{26}$ The region experiences a long rainy season from April to July, and short rains from October to November. Farming is the main economic activity and maize the staple food crop in this sparsely populated area. The typical local weaning food is the liquid maize porridge, 'uji'; a regular portion consists of about $8-10 \mathrm{~g}$ maize flour boiled in $100-150 \mathrm{~mL}$ water and sweetened with sugar.

In the catchment area of the Kikoneni health centre, we continuously recruited infants aged 5.5 months. We recruited 80 infants from March 2010 until September 2011, and randomly assigned them to receive either an MNP with or without $2.5 \mathrm{mg}$ iron as sodium iron EDTA (NaFeEDTA, $\pm 2.5 \mathrm{mgFeMNP}$, MixMe, DSM Nutritional Products Europe, Basel, Switzerland). We recruited 80 more infants from September 2011 to May 2012 and randomly assigned them to receive either an MNP with or without $12.5 \mathrm{mg}$ iron as ferrous fumarate $( \pm 12.5$ mgFeMNP, Sprinkles, Hexagon Nutrition, Mumbai). The composition of the MNPs is shown in table 1 . Inclusion criteria were an infant age of 5.5 months ( \pm 3 weeks), mother at least $\geq 15$ years of age, infant haemoglobin $(\mathrm{Hb}) \geq 70 \mathrm{~g} / \mathrm{L}$, and no maternal or infant chronic diseases. The MNPs were packed in group-coded sachets (containing one daily dose).

\section{Study design}

Prior to intervention, we conducted triangle sensory tests ${ }^{27}$ in local adults $(n=25$ per MNP). In these tests, the +FeMNPs were indistinguishable from the corresponding -FeMNPs, and their acceptability was high (data not shown).

Trained field workers instructed the mothers in cooking the 'uji' and its fortification with the MNPs. Further, the participating mothers were trained on the home collection of infant stool samples. Before starting the intervention with MNPs, we conducted a 2 -week run-in period to familiarise the families with the stool collection method and the introduction of the maize porridge.

Then, weekly for 4 months, we dispensed 7 MNP sachets and $2 \mathrm{~kg}$ of maize flour (Dola, Kitui Flour Mills, Mombasa, Kenya) 
Table 1 Composition of the two micronutrient powders, with and without iron*

\begin{tabular}{|c|c|c|c|c|}
\hline & $+2.5 \mathrm{mgFeMNP}$ & $-2.5 \mathrm{mgFeMNP}$ & +12.5 mgFeMNP & $-12.5 \mathrm{mgFeMNP}$ \\
\hline Iron, mg & 2.5 & - & 12.5 & - \\
\hline Zinc, mg & 2.5 & 2.5 & 5 & 5 \\
\hline Vitamin $A, \mu g$ & 100 & 100 & 300 & 300 \\
\hline Vitamin C, mg & 60 & 60 & 30 & 30 \\
\hline Vitamin $D, \mu g$ & 5 & 5 & - & - \\
\hline Copper, mg & 0.34 & 0.34 & - & - \\
\hline Tocopherol equivalent, mg & 5 & 5 & - & - \\
\hline lodine, $\mu \mathrm{g}$ & 30 & 30 & - & - \\
\hline Vitamin $\mathrm{K} 1, \mu \mathrm{g}$ & 30 & 30 & - & - \\
\hline Selenium, $\mu \mathrm{g}$ & 17 & 17 & - & - \\
\hline Thiamine, mg & 0.5 & 0.5 & - & - \\
\hline Riboflavin, mg & 0.5 & 0.5 & - & - \\
\hline Pyridoxine, mg & 0.5 & 0.5 & - & - \\
\hline Folic acid anhydrous, $\mu \mathrm{g}$ & 90 & 90 & - & - \\
\hline Niacinamide, mg & 6 & 6 & - & - \\
\hline Vitamin $B 12, \mu \mathrm{g}$ & 0.9 & 0.9 & - & - \\
\hline
\end{tabular}

${ }^{*}$ Amounts per $1 \mathrm{~g}$ powder.

directly to the participating mothers from six distribution points. We analysed triplicate samples of the maize flour for iron concentration by using atomic absorption spectroscopy, and for phytic acid 2829 at the ETH Zurich. During the weekly distribution, field workers collected the previous week's used and unused MNP sachets to assess compliance and recorded the infants' feeding history and the health status by using a multiplechoice questionnaire. If a mother reported any illness, the dedicated study nurse examined the child and recorded treated episodes of malaria, diarrhoea and respiratory tract infections (RTI). The study nurse used a forced-choice questionnaire to record treated cases of malaria, diarrhoea and RTI at baseline (covering the last 3 months) and after 4 months (covering the entire intervention period).

\section{Gut microbiome, short-chain fatty acids (SCFA) and faecal calprotectin}

Stool samples were collected at baseline, 3 weeks and 4 months. The mothers were provided with plastic diapers, containers, spatulas, Anaerocult sachets (Merck KGaA, Darmstadt, Germany) to generate an anaerobic environment, a falcon tube for the measurement of $8 \mathrm{~mL}$ water to wet the Anaerocult, and an illustrated pamphlet to reinforce the sampling instructions. The stool samples were collected in the morning, kept anaerobic, and aliquots were frozen at $-20^{\circ} \mathrm{C}$ the same day.

Full descriptions of the materials and methods used for the following are available in the online supplementary materials and methods: DNA extraction, gut microbiome analysis using $16 \mathrm{~S}$ rRNA pyrosequencing, targeted qPCR, and short-chain fatty acid (SCFA) measurements.

\section{Biochemical indicators}

At baseline, after 4 months $( \pm 12.5 \mathrm{mgFeMNP})$ and 6 months $( \pm 2.5 \mathrm{mgFeMNP})$, venous blood samples were drawn using heparin vacutainers and butterfly needles. Serum was separated and frozen on collection day.

The following parameters were determined, of which the full description is available in the online supplementary materials and methods: haemoglobin ( $\mathrm{Hb})$, zinc protoporphyrin to haem ratio (ZPP), serum ferritin (SF), soluble transferrin receptor
(sTfR) and C-reactive protein (CRP), serum hepcidin-25, body iron stores, serum IL-1 $\beta$, IL-2, IL-4, IL-5，IL-6，IL-8，IL-10, IFN $\gamma$, TNF $\alpha$, GM-colony-stimulating factor (CSF), IL-12 (p40/ p70) and IL-17. The following cut-offs were used: (1) anaemia: $\mathrm{Hb}<110 \mathrm{~g} / \mathrm{L},{ }^{30}$ (2) ID: body iron stores $<0 \mathrm{mg} / \mathrm{kg}^{31}$ and (3) inflammation: $\mathrm{CRP} \geq 4.1 \mathrm{mg} / \mathrm{L}$ (manufacturer's reference range).

\section{Anthropometric indicators}

At baseline and after 4 months, infant weight was recorded using a hanging scale (Salter 235-6S, $25 \mathrm{~kg} \times 100 \mathrm{~g}$; Salter Brecknell, UK) and length using a measurement board (Shorr Production, Olney, Maryland, USA). We calculated weight-forage (WAZ), height-for-age (HAZ), weight-for-height (WHZ) and BMI-for-age (Body Mass Index; BAZ) Z-scores using the WHO Anthro software. ${ }^{32}$

\section{Statistical analysis}

Based on previous studies on gut microbiota conducted by our group in schoolchildren ${ }^{22}$ and young adults, ${ }^{33}$ we estimated a sample size of 25-30 subjects in each group to detect a relevant difference of about $0.85 \mathrm{log}$ number of copies/g faeces in enterobacteria, lactobacilli and bifidobacteria, considering a SD of 1.09-1.37 for the three microbial groups, with $\alpha=0.05$ and $\beta=0.8$.

Longitudinal effects of iron on the gut microbiome (assessed by pyrosequencing) were assessed by comparing change over time, which was calculated by dividing the relative abundance of a taxon at 4 months or 3 weeks by the relative abundance of a taxon at baseline and a subsequent Mann-Whitney $U$ test (MWU) was done. Cross-sectional differences were compared by MWU. Estimated intervention effects of iron on gut microbiota (assessed by qPCR), iron status and anthropometrics were evaluated using univariate general linear models (GLM) with baseline values as covariates. Full descriptions of statistical methods and software used can be found in the online supplementary materials and methods.

\section{Ethical aspects}

This study was approved by the ethics and research committees of the Kenyatta National Hospital/ University of Nairobi (P167/ 
6/2009 and P168/05/2011), and the ETH Zurich (EK 2009-N-53). Caregivers of the infants gave written informed consent. A data safety monitoring board, including a paediatrician and a nutritionist, performed safety monitoring for the study.

\section{RESULTS}

Our estimated sample size was 100-120 infants, and we enrolled 115 infants into the study (figure 1 , trial profile). We originally recruited 160 infants, but during the 2-week run-in period, 21 infants were excluded because they had received antibiotics in the previous 2 months, and 24 infants did not provide an adequate baseline stool sample. During the study, a further 11 infants were excluded because they received antibiotics, or were unable to provide adequate stool samples. Use of antibiotics was unexpectedly high in this setting; this was the local standard of care. Due to a labelling error at the factory in a second batch of the $\pm 2.5 \mathrm{mgFeMNP}$ sachets, three infants received mixed treatments and were excluded. Therefore, the final analysis was completed on 101 infants: 28 from $+2.5 \mathrm{mgFeMNP}, 21$ from $-2.5 \mathrm{mgFeMNP}, 26$ from $+12.5 \mathrm{mgFeMNP}$, and 26 from $-12.5 \mathrm{mgFeMNP}$.

Baseline prevalence of anaemia, iron deficiency (negative body iron stores) and systemic inflammation were $67.3 \%$, $25.5 \%$ and $29.7 \%$, respectively. Nearly all infants were still being breastfed $(99.0 \%)$, but $80.2 \%$ had already been introduced to complementary foods, predominantly 'uji' starting, on average, at 4 months of age. Compliance with the sachets was 99.4\% for $\pm 2.5 \mathrm{mgFeMNP}$ and $96.2 \%$ for $\pm 12.5 \mathrm{mgFeMNP}$. The native iron and phytic acid concentrations in the maize flour were $1.15 \pm 0.06 \mathrm{mg}$ and $310 \pm 20 \mathrm{mg}$ per $100 \mathrm{~g}$, respectively.

\section{Baseline infant gut microbiome and its development over time}

Totally, 934853 bacterial 16S rRNA sequences were analysed by pyrosequencing. At baseline, the infant gut microbiome consisted of the phyla Actinobacteria (64.3\% of reads), Firmicutes (22.4\%; including 4.7\% Lactobacillus), Bacteroidetes (8.9\%; including $3.6 \%$ Bacteroides and $4.3 \%$ Prevotella), and
Proteobacteria (4.1\%; including 3.3\% Escherichia/Shigella); and was highly dominated by the family of Bifidobacteriaceae, contributing $63.0 \%$ of the total $16 \mathrm{~S}$ rRNA (figure 2). There was no significant effect of baseline anaemia status on phylogenetic diversity among the infants (data not shown). However, an exploratory analysis revealed significant differences in taxa between anaemic and non-anaemic infants at baseline: nonanaemic infants harboured lower abundances of Prevotella $(2.0 \%$ vs $4.5 \%, p=0.014)$, whereas, they showed higher abundances of Actinomycetales $(0.14 \%$ vs $0.09 \%, p=0.004)$ and Streptococcus $(6.3 \%$ vs 3.9\%, $\mathrm{p}=0.023)$ (figure 3 ).

The phylogenetic diversity in all stool samples increased after 3 weeks $(p=0.004)$ and further increased to 4 months $(p=0.005$, see online supplementary figure S1). Redundancy analysis throughout the study found: (1) a strong interindividual variation of the gut microbial composition $(p=0.001)$; (2) a significant difference in the gut microbiome of anaemic and non-anaemic infants $(\mathrm{p}=0.031)$ and $(3)$ a significant difference in the gut microbiome of infants in the two MNP trials $( \pm 2.5 \mathrm{mgFeMNP}$ and $\pm 12.5 \mathrm{mgFeMNP}, \mathrm{p}=0.015)$. Furthermore, strong time-specific signatures were found $(p=0.001)$. Gender, season in which the intervention started, and the starting date of complementary feeding did not significantly affect the gut microbiome.

Significant changes over time in the infants gut microbiome composition of the -FeMNP group from baseline (6 months old) to endpoint (10 months old) are illustrated in online supplementary figure S2. These changes involved an increase in Faecalibacterium and Prevotella, and a decrease in Enterobacteriaceae $(\mathrm{p}=0.010, \mathrm{p}=0.006, \mathrm{p}=0.002$, respectively).

\section{Composition of the infant gut microbiome during iron fortification}

The phylogenetic diversity of the gut microbiome was not significantly modified by + FeMNP versus -FeMNP (see online supplementary figure S1). Differences in the gut microbiome at endpoint for the combined \pm FeMNP groups and the separate analysis of the $\pm 2.5 \mathrm{mgFeMNP}$ and $\pm 12.5 \mathrm{mgFeMNP}$ are shown in figure $4 \mathrm{~A}-\mathrm{C}$. The changes over time in the relative abundance of a taxon between the \pm FeMNP groups and the $\pm 2.5 \mathrm{mgFeMNP}$ and $\pm 12.5 \mathrm{mgFeMNP}$ are shown in figure $5 \mathrm{i}-\mathrm{vi}$.

Figure 1 Trial profile. 


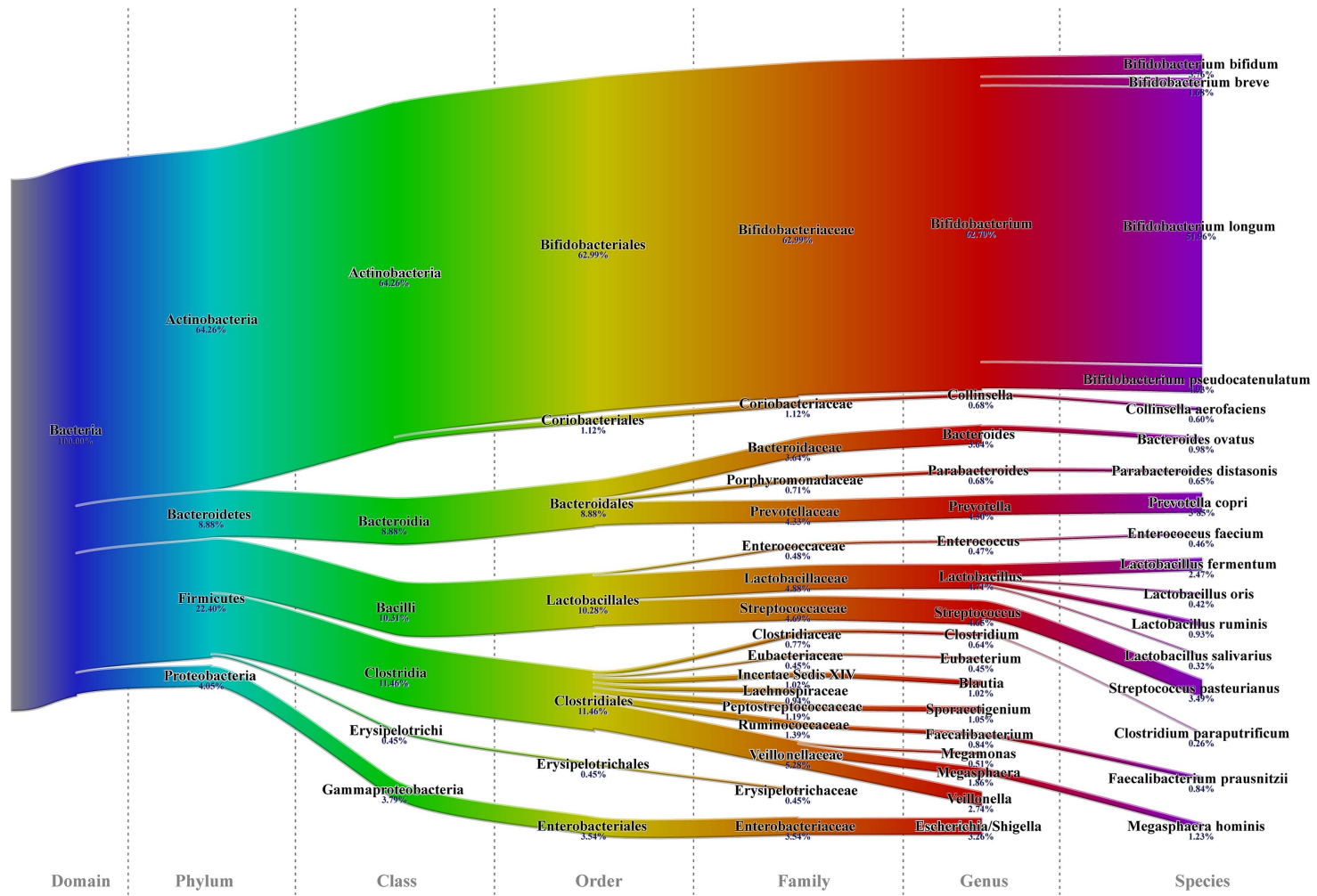

Figure 2 Baseline gut microbiome of the 6 month-old Kenyan infants enumerated by 16S rRNA pyrosequencing ( $n=101$ ). The fraction of 16S rRNA reads (in \%) attributed to specific taxonomic level is given below the taxon name.

Comparing +FeMNP versus -FeMNP, there were significantly higher abundances of the genera Clostridium, and Escherichial Shigella $(\mathrm{p}=0.033, \mathrm{p}=0.010$, respectively), and a trend towards lower abundances of the genus Bifidobacterium $(\mathrm{p}=0.085)$ in the + FeMNP group at endpoint (figure 4A). Additionally, the change over time in relative abundances (4 months vs baseline) was significantly different in +FeMNP versus -FeMNP for Firmicutes, Escherichia/Shigella, and Clostridium $(\mathrm{p}=0.034$, $\mathrm{p}=0.030, \mathrm{p}=0.048$, respectively; figure 5i-iii). Comparing $+2.5 \mathrm{mgFeMNP}$ with $-2.5 \mathrm{mgFeMNP}$, there were significantly higher abundances of the genera Escherichia/Shigella $(\mathrm{p}=0.044)$ and a trend towards higher in Roseburia $(\mathrm{p}=0.083)$ in +2.5 mgFeMNP versus $-2.5 \mathrm{mgFeMNP}$ at endpoint (figure 4B). Additionally, the change over time in relative abundances
(4 months vs baseline) was significantly different in $+2.5 \mathrm{mgFeMNP}$ compared with $-2.5 \mathrm{mgFeMNP}$ for Escherichia/Shigella $\quad(\mathrm{p}=0.034$; figure 5iv $)$ Comparing $+12.5 \mathrm{mgFeMNP}$ with $-12.5 \mathrm{mgFeMNP}$, there were significantly higher abundances of Firmicutes $(p=0.018)$, Bacteroides $(p=0.045)$, a trend towards higher abundances of Clostridium species $(p=0.052)$ and Escherichia/Shigella $(p=0.067)$, and significantly lower abundance of Bifidobacterium $(\mathrm{p}=0.047)$ in $+12.5 \mathrm{mgFeMNP}$ versus $-12.5 \mathrm{mgFeMNP}$ at endpoint (figure 4C). Additionally, the change over time in relative abundances (4 months vs baseline) was significantly higher in $+12.5 \mathrm{mgFeMNP}$ compared with $-12.5 \mathrm{mgFeMNP}$ for Firmicutes $(p=0.046$; figure $5 v)$ and showed a significantly larger decrease for Bifidobacterium ( $\mathrm{p}=0.049$, figure 5vi).
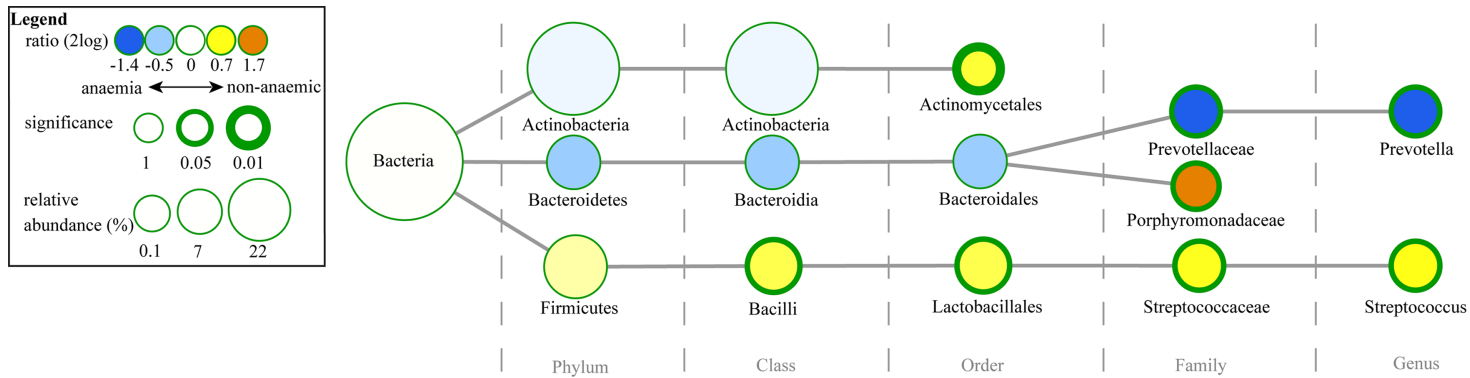

Figure 3 Differences in baseline gut microbiome composition in anaemic versus non-anaemic 6 month-old Kenyan infants. Nodes represent taxa; edges link the different taxonomic levels. The fold increase is calculated as the 2 log of the ratio of the relative abundance in anaemic and non-anaemic ( $0=$ no difference between anaemia status, $1=$ twice as abundant in non-anaemic). The significance is expressed as the $p$ value of a Mann-Whitney $\mathrm{U}$ test of the anaemic and non-anaemic infant samples. The node-size corresponds to the relative abundance. Taxa (ie, nodes) were included in this visualisation if they met the following criteria: all samples together have an average relative abundance of $>0.1 \%$ for the taxon, and the study groups have a fold-difference of at least 0.1 with a significance of $p<0.05$ or the taxon has a child (ie, more specific taxonomic classification) meeting the criteria. 

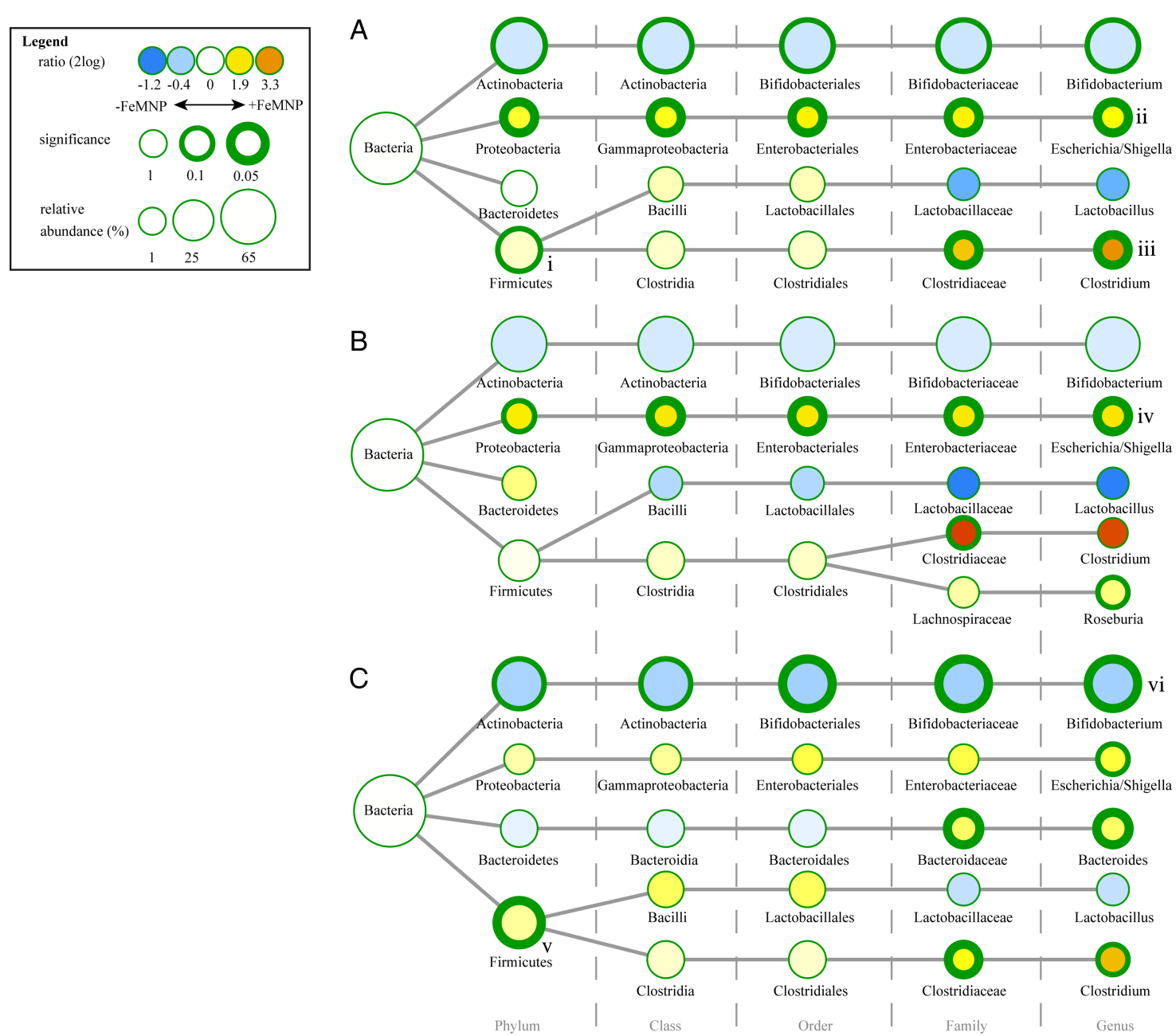

Figure 4 Differences in gut microbial composition after 4 months in Kenyan infants receiving iron-containing micronutrient powders (+FeMNP) versus no-iron micronutrient powders (-FeMNP). (A) +FeMNP vs -FeMNP; (B) +2.5 mgFeMNP vs -2.5 mgFeMNP; (C) +12.5 mgFeMNP vs $-12.5 \mathrm{mgFeMNP}$. Nodes represent taxa; edges link the different taxonomic levels. The fold increase is calculated as the 2 log of the ratio of the relative abundance in +FeMNP and $-\mathrm{FeMNP}(0=$ no difference between group, $1=$ twice as abundant in $+\mathrm{FeMNP}$, etc.). The significance is expressed as the $\mathrm{p}$ value of a Mann-Whitney $\mathrm{U}$ test. The node-size corresponds to the relative abundance (in \%). Taxa displayed were selected based on the list of targets of our primary interest.

Comparing + FeMNP versus -FeMNP by qPCR analysis, revealed a borderline significant effect of iron on enterobacteria $(\mathrm{p}=0.061)$, with higher concentrations in +FeMNP $(8.9 \pm 0.3$ $\log$ number of gene copies/g faeces) versus -FeMNP (8.0 \pm 0.4$)$ at endpoint. Furthermore, there was a significant treatment effect on Roseburia species/Eubacterium rectale $(\mathrm{p}=0.020)$, with lower concentrations in +FeMNP $(4.4 \pm 0.4)$ versus -FeMNP $(5.8 \pm 0.4)$. In the separate analyses of the two MNPs, a significant treatment effect was found on Roseburia species/E rectale in $\pm 12.5 \mathrm{mgFeMNP}(\mathrm{p}<0.0001)$, with lower concentrations in $+12.5 \mathrm{mgFeMNP}(3.2 \pm 0.4)$ versus $-12.5 \mathrm{mgFeMNP}(6.2 \pm 0.5)$ at endpoint; while a significant treatment effect was seen for Eubacterium hallii in $\pm 2.5 \mathrm{mgFeMNP}(\mathrm{p}=0.009)$, with higher concentrations in $+2.5 \mathrm{mgFeMNP}(8.4 \pm 0.6)$ versus $-2.5 \mathrm{mgFeMNP}(7.1 \pm 0.5)$ at endpoint.

Examining the effect of iron on pathogenic gut microbiota in all analysed stool samples, we detected Bacillus cereus in 39.5\% (mean in detected samples: 4.6 log copies/g faeces (range: 3.3-7.2)), Staphylococcus aureus in $65.4 \%$ (6.6 (3.0-10.0)), Clostridium difficile in $56.5 \%$ (7.2 (3.4-10.4)), members of the Clostridium perfringens group in 89.7\% (7.7 (3.1-10.4)), Salmonella in $22.4 \%(5.7(4.4-7.9))$ and Vibrio cholera in $0 \%$.
Further, we detected enteropathogenic E. coli (EPEC) in 65.0\% (mean in detected samples: 6.0 log copies/g faeces (range: $3.2-$ 9.3)), enterotoxigenic E. coli producing heat-labile toxin (ETEC LT) in $49.2 \%(6.0(3.0-10.5))$, ETEC producing heat-stable toxin (ETEC ST) in 7.0\% (5.7 (3.5-8.7)), enterohaemorrhagic E. coli producing shiga-like toxin 1 (EHEC stx1) in $9.6 \%(4.7$ (3.4-7.4)), and EHEC stx2 in 8.5\% (4.6 (3.6-6.3)). There was a significant treatment effect on the sum of the pathogenic E. coli at endpoint $(\mathrm{p}=0.029)$, with higher concentration in the + FeMNP $(6.0 \pm 0.5 \log$ numbers of copies/g faeces) versus - FeMNP $(4.5 \pm 0.5)$. In the separate analyses of the two MNPs, there was a significant treatment effect on the sum of the pathogenic E. coli in $\pm 2.5 \mathrm{mgFeMNP}$ at midpoint $(\mathrm{p}=0.012)$, and at endpoint $(p=0.043)$, with transient lower concentrations in the $+2.5 \mathrm{mgFeMNP}(4.9 \pm 0.7)$ versus $-2.5 \mathrm{mgFeMNP}(7.0 \pm 0.5)$ at midpoint and higher concentrations in $+2.5 \mathrm{mgFeMNP}(6.5$ $\pm 0.6)$ versus $-2.5 \mathrm{mgFeMNP}(4.1 \pm 0.8)$ at endpoint. No effect of iron on pathogenic E. coli could be detected in the $\pm 12.5 \mathrm{mgFeMNP}$. The increase in the sum of pathogenic E. coli through iron fortification was significant in infants with baseline iron deficiency $(p=0.012)$, but not in infants who were iron sufficient $(p=0.327)$. 

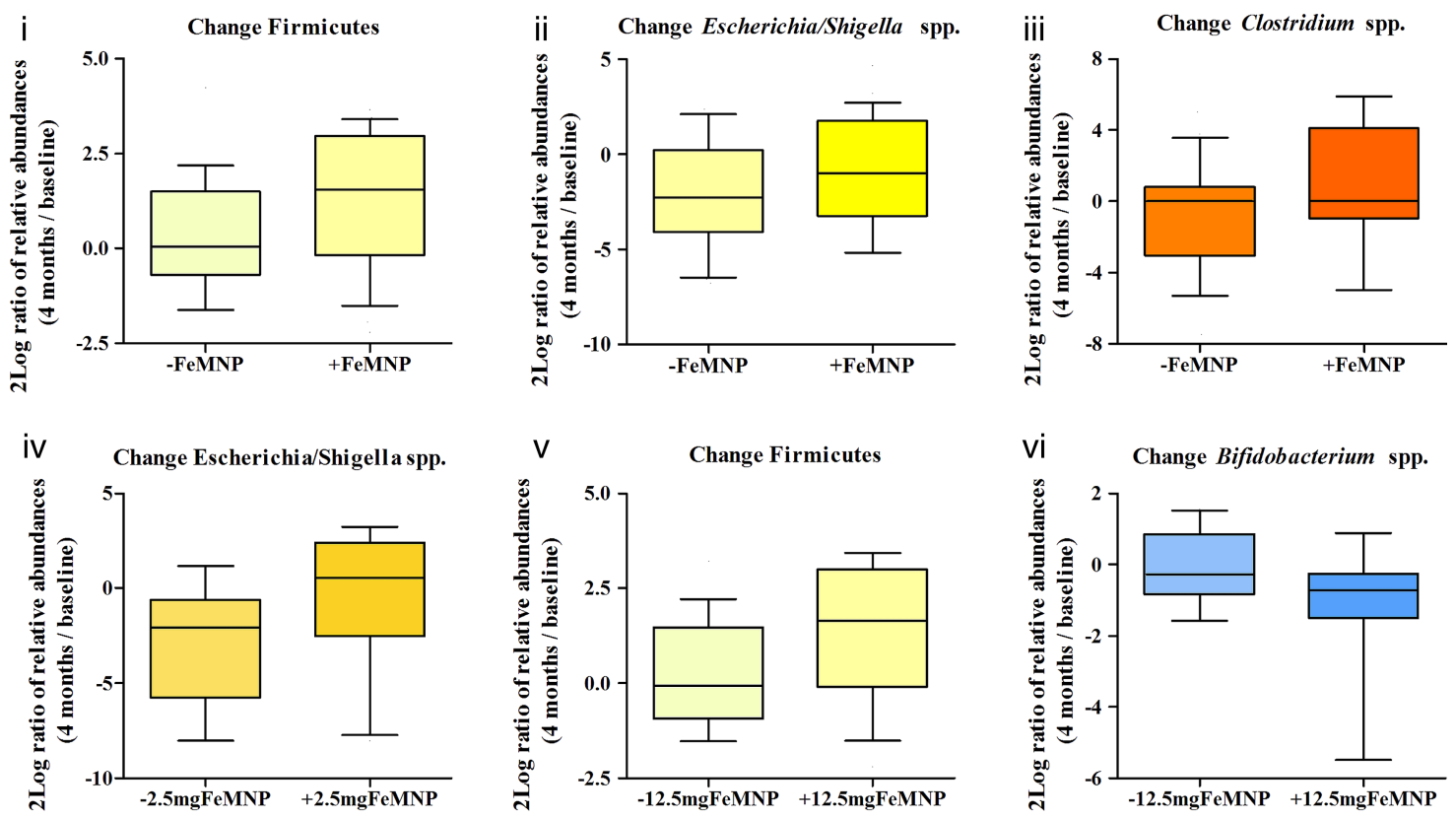

Figure 5 Change from baseline to 4 months in taxa that differed significantly between +FeMNP and - FeMNP infants at 4 months. The box plots (i-vi) report on significant changes over time from baseline to endpoint (i: $p=0.0 .034, i i: p=0.030$, iii: $p=0.048, i v: p=0.034, v$ : $p=0.046$, vi: $p=0.049$ ) of taxa being different in groups at endpoint (corresponding roman numerals and colours in figure 4). Boxplots of 2 log ratios ( $0=$ no difference for the two time points, $1=$ twice as abundant at 4 months, etc.) are displayed with the 10-90th percentiles.

Figure 6A-D shows the ratio of abundances of enterobacteria to bifidobacteria and lactobacilli analysed by pyrosequencing and qPCR. Using pyrosequencing, the ratio of the relative abundances of enterobacteria to bifidobacteria changed significantly over time from baseline to endpoint in the +FeMNP versus - FeMNP ( $p=0.020$; figure 6A). At endpoint, the enterobacteria to bifidobacteria ratio was significantly higher in the +FeMNP versus -FeMNP $(p=0.004)$; similarly, in the separate analysis of the MNPs, the enterobacteria to bifidobacteria ratio was significantly higher in $+2.5 \mathrm{mgFeMNP} \quad(\mathrm{p}=0.030)$ and in +12.5 mgFeMNP $(p=0.049)$, compared to the respective control groups at endpoint. The qPCR analysis confirmed this, showing a significant effect on the ratio of enterobacteria to bifidobacteria in +FeMNP compared with -FeMNP group at endpoint $(p=0.008$, figure $6 \mathrm{~B})$; with a trend towards a significant effect in the separate analysis of $\pm 2.5 \mathrm{mgFeMNP}(\mathrm{p}=0.053)$ and $\pm 12.5 \mathrm{mgFeMNP}(\mathrm{p}=0.098)$. Using pyrosequencing, the ratio of the relative abundance of enterobacteria to lactobacilli in +FeMNP did not change over time from baseline to endpoint, after a temporary decrease at 3 weeks $(p=0.018)$ in +FeMNP versus -FeMNP; however, at endpoint, the ratio of enterobacteria to lactobacilli was significantly higher in + FeMNP $(\mathrm{p}=0.023$; figure $6 \mathrm{C})$. Using $\mathrm{qPCR}$, a similar trend towards a higher ratio of enterobacteria to lactobacilli was detected at endpoint in +FeMNP compared with -FeMNP $(p=0.062$, figure 6D). Furthermore, pyrosequencing analysis of $\pm 2.5 \mathrm{mgFeMNP}$ and $\pm 12.5 \mathrm{mgFeMNP}$ separately showed a borderline significantly higher ratio of enterobacteria to lactobacilli in $+12.5 \mathrm{mgFeMNP}$ versus $-12.5 \mathrm{mgFeMNP}(\mathrm{p}=0.055)$ at endpoint, with a temporary decrease after 3 weeks $(p=0.021)$; but did not differ at any time point in $+2.5 \mathrm{mgFeMNP}$ versus $-2.5 \mathrm{mgFeMNP}$.

\section{Faecal calprotectin, SCFAs, iron status and systemic} inflammation

At endpoint, intestinal inflammation, assessed by faecal calprotectin, was significantly higher in infants receiving +FeMNP
$(229.2 \pm 1.9 \mu \mathrm{g} / \mathrm{g})$ versus -FeMNP $(123.3 \pm 2.1 \mu \mathrm{g} / \mathrm{g}, \mathrm{p}=0.002)$ In the separate analyses of the MNPs, faecal calprotectin values were significantly elevated in $+12.5 \mathrm{mgFeMNP}(248.9 \pm 2.2 \mu \mathrm{g} /$ g) versus $-12.5 \mathrm{mgFeMNP}(102.5 \pm 2.2 \mu \mathrm{g} / \mathrm{g}, \mathrm{p}=0.008)$, but were not significantly higher in $+2.5 \mathrm{mgFeMNP}(215.6$ $\pm 1.6 \mu \mathrm{g} / \mathrm{g})$ versus $-2.5 \mathrm{mgFeMNP}(165.6 \pm 1.8 \mu \mathrm{g} / \mathrm{g}, \mathrm{p}=0.164$, figure 7). The increase in faecal calprotectin through iron fortification was significant in infants who were iron sufficient at baseline $(p=0.0002)$, but not in infants with iron deficiency $(p=0.912)$. There were no significant correlations of faecal calprotectin with the overall gut microbiome composition assessed by pyrosequencing. The qPCR data revealed intrasample correlations of faecal calprotectin with none of the commensal bacteria, but with the sum of pathogenic E. coli $(\mathrm{p}=0.011$, $\mathrm{t}=0.177)$, and enterobacteria $(\mathrm{p}=0.008, \mathrm{t}=0.201)$, and members of the C. perfringens group $(p=0.007, t=-0.206)$ at 3 weeks, and $\operatorname{EPEC}(p=0.024, t=0.190)$ at 4 months. There were no significant differences in faecal acetate, propionate, or butyrate concentrations between +FeMNPs and -FeMNPs during the intervention (see online supplementary table S2).

There was a significant treatment effect of $+12.5 \mathrm{mgFeMNP}$ versus $-12.5 \mathrm{mgFeMNP}$ on body iron $(\mathrm{p}=0.001)$, SF $(p=0.004)$, sTfR $(p=0.008), \operatorname{ZPP}(p=0.039)$ and a trend towards an effect on hepcidin-25 ( $p=0.052$, see online supplementary table S3). By contrast, there was no significant treatment effect of $+2.5 \mathrm{mgFeMNP}$ versus $-2.5 \mathrm{mgFeMNP}$ on any iron status indicator or hepcidin-25. There was no treatment effect of either +FeMNP on serum CRP (see online supplementary table S3) or serum IL-1 $\beta$, IL-2, IL-4, IL-5, IL-6, IL-8, IL-10, IL-17, IFN $\gamma$, TNF $\alpha$ or GM-CSF (data not shown). There was a significant treatment effect of $+2.5 \mathrm{mgFeMNP}$ versus $-2.5 \mathrm{mgFeMNP}$ on IL-12, which was higher in $+2.5 \mathrm{mgFeMNP}$ (523.6 $\pm 1.5 \mu \mathrm{g} / \mathrm{g}$ vs $431.9 \pm 1.4 \mu \mathrm{g} / \mathrm{g}, \mathrm{p}=0.028)$.

\section{Growth}

There was no significant treatment effect of iron on weight gain. However, we identified a significant increase of linear growth 

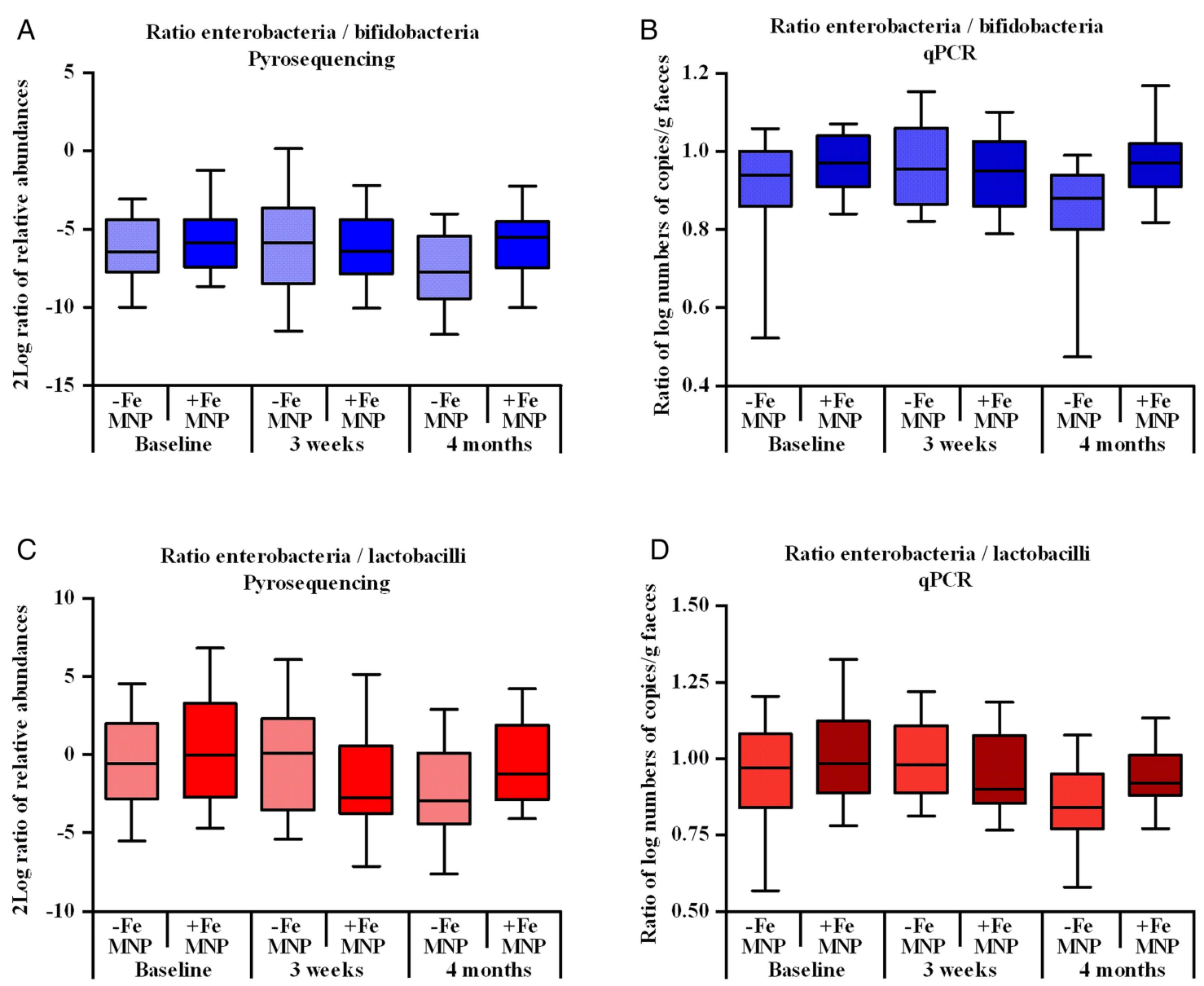

Figure 6 Ratio of infant enterobacteria to bifidobacteria at baseline, 3 weeks and 4 months, comparing +FeMNP and -FeMNP assessed by: (A) pyrosequencing (log2 ratio of the relative abundance); and (B) QPCR (ratio of log numbers of copies/g faeces). Ratio of infant enterobacteria to lactobacilli assessed by: (C) pyrosequencing; and (D) qPCR. Boxplots are displayed with the 10-90th percentiles. Values differed significantly between groups (+FeMNP and -FeMNP): (A) the change over time from baseline to endpoint in ratios was significantly different between groups $(p=0.020)$; at endpoint, the ratios were significantly higher in +FeMNP versus -FeMNP $(p=0.004)$. (B) the ratios were significantly different between groups at 4 months using general linear models (GLM) and adjusted for baseline differences $(p=0.008)$. (C) the change over time from baseline to 3 weeks in ratios was significantly different $(p=0.018)$, but the change over time from baseline to endpoint was not different between +FeMNP versus -FeMNP; at endpoint the ratios were significantly higher in +FeMNP versus -FeMNP at endpoint $(p=0.023)$. (D) the ratios were borderline significantly different between groups at 4 months using GLM and adjusted for baseline differences $(p=0.062)$.

in +FeMNPs $(70.2 \pm 1.1 \mathrm{~cm}$ vs $68.5 \pm 1.1 \mathrm{~cm}$ at endpoint, $\mathrm{p}=0.017$, see online supplementary table S3). This effect was significant in the infants of $\pm 12.5 \mathrm{mgFeMNP}(70.2 \pm 1.1 \mathrm{~cm}$ vs $68.1 \pm 1.1 \mathrm{~cm}$ at endpoint, $\mathrm{p}=0.011)$, but not in $\pm 2.5 \mathrm{mgFeMNP}$. Linear growth was not correlated to gut microbiota or faecal calprotectin.

\section{Morbidity}

On enrolment, $19.2 \%$ of mothers reported a treated episode of diarrhoea in their infant during the previous 3 months, $29.7 \%$ a treated RTI, and $3.8 \%$ a treated malaria episode. During the intervention, incidences of treated RTI and malaria did not significantly differ between +FeMNP versus -FeMNP. However, there was a trend towards a greater incidence of treated episodes of diarrhoea in $+12.5 \mathrm{mgFeMNP}$ versus $-12.5 \mathrm{mgFeMNP}$ : $27.3 \%(n=6 / 22)$ versus $8.3 \%(n=2 / 24, p=0.092)$.

\section{DISCUSSION}

This study shows that in breastfed, 6-month-old infants from rural Africa, the gut microbiome is dominated by Bifidobacteriaceae, but harbours many gram-negative and grampositive pathogens. Our findings indicate that the iron in MNPs favours growth of several of these potentially pathogenic subgroups. At the same time, iron decreases abundances of bifidobacteria, shifting gut microbial balance away from beneficial 'barrier' strains towards a potentially more pathogenic profile. This is accompanied by an increase in intestinal inflammation. Our findings in Kenyan infants are comparable with those of a recent controlled trial in school-aged children in Côte d'Ivoire receiving iron-fortified biscuits containing $20 \mathrm{mg}$ iron/day as electrolytic iron for 6 months. ${ }^{22}$ The expected absorption of electrolytic iron in that setting was $<5 \%$, and there was no decrease in anaemia or iron deficiency in the iron-fortified group. Although the study was a secondary analysis, only qPCR methods were used, and the authors did not report increases in specific pathogens; it did demonstrate that iron fortification can extensively modify the gut microbiota, increasing enterobacteria and decreasing lactobacilli, along with increased intestinal inflammation measured by faecal calprotectin. The differences between these two studies are likely due to age-related differences in the gut microbiota between infants and older children, but may also reflect differences in methods used to characterise the gut microbiome, differences in geographic setting and, possibly, differences in the iron compound and/or dose given. 


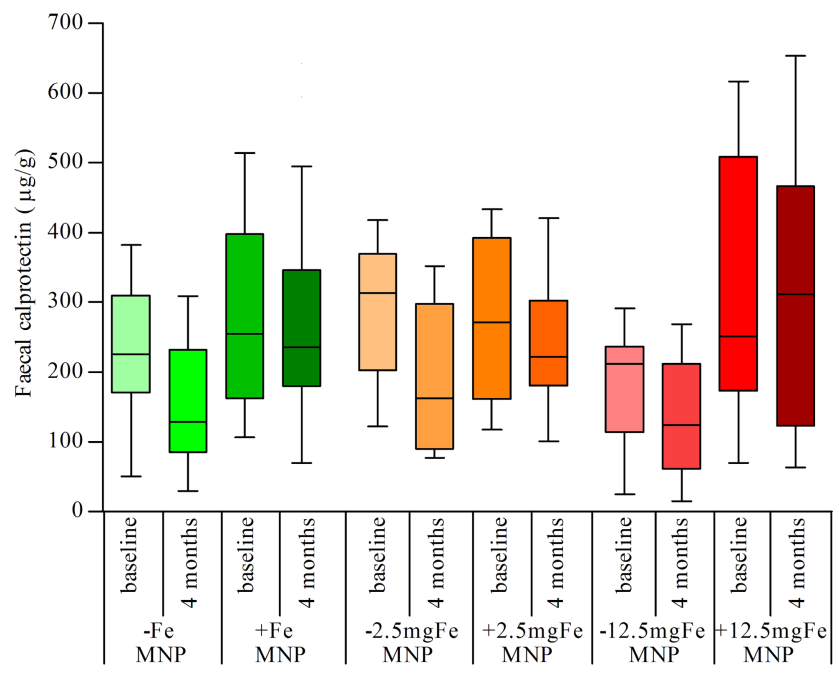

Figure 7 Infant faecal calprotectin concentrations at baseline and 4 months in the pooled data from the -FeMNP versus +FeMNP groups, in the $-2.5 \mathrm{mgFeMNP}$ versus $+2.5 \mathrm{mgFeMNP}$, and in the

$-12.5 \mathrm{mgFeMNP}$ versus $+12.5 \mathrm{mgFeMNP}$. Values differed significantly over the trial between the \pm 12.5 mgFeMNP groups $(p=0.008)$ and the pooled MNP groups ( \pm FeMNP, $p=0.002)$, using general linear models and baseline variables as covariates. Boxplots are displayed with the 10-90th percentiles.

A recent high-dose iron supplementation trial in low to middle-income South African schoolchildren residing in an area with an improved water supply and a lower risk of contaminated food, ${ }^{34}$ supports the suggestion that environmental variables modulate the effects of iron on intestinal inflammation and gut microbiota. Despite the high iron dose, there were no measurable effects on intestinal inflammation measured by faecal calprotectin, nor on gut microbiota evaluated by qPCR. Overall, these data suggest that the effects of supplemental or fortification iron on the gut microbiota and intestinal inflammation in children are more pronounced in settings where hygiene standards are low and the microbiome is likely to be populated by opportunistic enteropathogens.

Iron fortification in the present study, and in the Ivorian study, ${ }^{22}$ favoured growth of enterobacteria over bifidobacteria and/or lactobacilli, and this could be due to their different iron requirements and metabolism. Many pathogenic enterobacteria require iron acquisition for bacterial virulence ${ }^{3536}$ and/or gastrointestinal tract colonisation. ${ }^{37}$ Only few bacteria do not require iron, of which lactobacilli is the major group. ${ }^{17}$ Lactobacilli do not produce siderophores, and their growth is similar in media with and without iron. ${ }^{38}$ Bifidobacterium breve, an important bifidobacteria species in breastfed infants, can sequester luminal iron using a divalent metal permease, ${ }^{39} 40$ but the majority of bifidobacteria do not produce siderophores or other active iron carriers. Abundant bifidobacteria, lactobacilli and other beneficial bacteria in the colon provide an important 'barrier effect' against colonisation and invasion by pathogens. ${ }^{17} 4142$ Our findings suggest that +FeMNPs weaken this protective effect. If + FeMNPs promote expansion of enterobacteria, this may be important because abundances of closely related species can predict susceptibility to intestinal colonisation by pathogenic bacteria. ${ }^{43}$ In our study, this effect may have encouraged colonisation by potentially pathogenic members of the genus Escherichia/Shigella evidenced by the higher abundances of this genus in the +FeMNP groups at endpoint and, in particular of the five pathogenic E. coli subgroups (EPEC, ETEC LT, ETEC ST, EHEC stx1, and stx2).
Calprotectin is a calcium-binding and zinc-binding protein found in the cytosol of neutrophils, monocytes and activated macrophages. ${ }^{44}$ Faecal calprotectin levels mainly reflect migration of neutrophils into the gut mucosa, and is a non-specific marker of intestinal inflammation elevated in children with gastroenteritis. ${ }^{45}$ Faecal calprotectin is markedly higher during infancy than in later childhood. ${ }^{46}$ In healthy Ugandan children, median faecal calprotectin were $278 \mu \mathrm{g} / \mathrm{g}$ at 3-6 months, $183 \mu \mathrm{g} / \mathrm{g}$ at $6-12$ months of age, and fell to $28 \mu \mathrm{g} / \mathrm{g}$ at school age. ${ }^{47}$ We used a different assay than the Ugandan study, but found comparable levels of faecal calprotectin at baseline. In -FeMNP groups, faecal calprotectin decreased as expected over the 4-month intervention, while in +FeMNP groups, and particularly in $+12.5 \mathrm{mgFeMNP}$, levels remained significantly elevated. In the Ivorian fortification study, where school-age children received $\approx 9 \mathrm{mg}$ of iron per day, faecal calprotectin increased sharply and was correlated with the increase in gut enterobacteria. ${ }^{22}$ Although we found no significant difference in systemic concentrations of most cytokines, circulating IL-12 concentrations were significantly higher in $+2.5 \mathrm{mgFeMNP}$ versus $-2.5 \mathrm{mgFeMNP}$ at endpoint. Gut microbial antigens can stimulate secretion of IL-12 by gut macrophages and induce development of Th1 cells. ${ }^{48}$ Thus, in our study, IL-12 secretion may have been triggered by invasive pathogens or dysbiosis resulting from increased luminal iron; notably, in +2.5 mgFeMNP versus -2.5 mgFeMNP, the Escherichia/Shigella taxon was significantly higher at endpoint, an effect not seen in +12.5 mgFeMNP. Furthermore, dietary iron has shown to increase intestinal inflammation in this study and others ${ }^{22} 49$ through different potential pathways, such as neutrophil infiltration, lipid peroxidation, NF- $\mathrm{KB}$ activation and proinflammatory cytokines. ${ }^{49}$ Electron acceptors generated as by-products of host inflammatory response have been proposed to favour facultative anaerobes, in particular, enterobacteria. ${ }^{50}$

The +FeMNPs we used in this study contained iron as two different forms, ferrous and ferric, and the ferric iron was bound to a chelator (EDTA). Our data do not clarify whether the form of dietary iron is a potential determinant of the changes induced in the gut microbiota, as the relative concentration of ferrous versus ferric iron in the human colon resulting from dietary iron ingestion is unknown. However, even if different forms of iron are ingested, it is likely that most of the unabsorbed dietary iron that enters the colon is in the oxidised, ferric $\left(\mathrm{Fe}^{+3}\right)$ form, mainly as ferric oxides or hydroxides. ${ }^{51}$ In the colon, low-oxygen tension would favour the reduction of ferric to ferrous $\left(\mathrm{Fe}^{+2}\right)$ iron. Systems for acquisition of ferrous and ferric iron have been identified in many gastrointestinal pathogens. Although ferric ion has a solubility of only $10^{-17} \mathrm{M}$ at $\mathrm{pH} 7$, and bacteria generally require iron at around $10^{-7}-10^{-5} \mathrm{M}$ to achieve optimal growth, ${ }^{14}$ bacteria can reduce ferric iron to the more soluble ferrous form and/or use extracellular ferric iron chelators, such as siderophores, as solubilising agents prior to uptake. ${ }^{52}$ Many enteric pathogens, including Salmonella species and Escherichial Shigella species, ${ }^{15}$ take up iron-siderophore complexes via specific outer membrane receptors. Most enteric gram-negative bacteria also have active transport systems for ferrous iron, ${ }^{14}$ and iron transporter FeoB-mediated ferrous iron acquisition may increase virulence. ${ }^{36}$

In our study, the ferrous fumarate-containing $+12.5 \mathrm{mgFeMNP}$ tended to cause greater modifications of the gut microbiome and more intestinal inflammation; this was likely due to the fact that its iron dose was fivefold that of the chelated ferric iron in the $+2.5 \mathrm{mgFeMNP}$. However, it is not clear from our findings if the higher iron dose was more 
detrimental: although $+12.5 \mathrm{mgFeMNP}$ resulted in lower abundances of Bifidobacterium, and higher levels of faecal calprotectin, as well as a trend towards higher rates of treated diarrhoea, the effect on Escherichia/Shigella, the ratio of enterobacteria to bifidobacteria, and on the increase of pathogenic E. coli was stronger with $+2.5 \mathrm{mgFeMNP}$. Therefore, our data do not clarify whether the lower iron dose MNP would have a better safety profile.

In accordance with previous studies on human gut microbiota, ${ }^{53-56}$ the four dominant phyla in our infants at baseline were Actinobacteria (63\%, mainly Bifidobacteriaceae), Firmicutes (22\%), Bacteroidetes (9\%), and Proteobacteria (4\%). We found more distinct treatment effects after 4 months than after 3 weeks, likely due to the rapid fluctuations in the gut microbiome at 3 weeks caused by the introduction of the complementary feeding. ${ }^{20}$ This finding underscores the importance of allowing the gut microbiome time to adapt to changes in diet when evaluating an intervention and comparing different trials. Our data from the gut microbiome of African infants are comparable with those reported in Dutch infants using qPCR methods that found similar abundances of bifidobacteria, E. coli, and lactobacilli. ${ }^{18}$ However, by contrast, many of our infants carried enteropathogens, with over half the faecal samples containing C. difficile, S. aureus, members of the C. perfringens group, enteropathogenic and/or enterotoxigenic E. coli, and nearly one in four harbouring B. cereus and/or Salmonella. Our data also suggest the baseline gut microbiome of anaemic versus non-anaemic infants may differ, as may their response to +FeMNPs. Anaemic infants showed higher Prevotella (Bacteroidetes) and lower Actinomycetales (Actinobacteria) and Streptococcus (Firmicutes) abundances than non-anaemic infants. A previous study comparing the gut microbiome of older children from Burkina Faso and Italy found higher concentrations of Bacteroidetes (especially Prevotella and Xylanibacter) and lower concentrations of Firmicutes and Escherichia/Shigella in the Burkinabe than Italian children. ${ }^{53}$ Thus, our findings suggest higher rates of anaemia in African children may contribute to differences observed in gut microbiota in African versus European children.

In rats, the availability of colonic iron may modulate gut microbiota metabolites and, in particular, production of caecal SCFAs. ${ }^{57}$ This could have important gut modulatory effects, as SCFAs have been reported to inhibit growth or reduce concentrations of Salmonella enteritidis, Salmonella enterica serovar Typhimurium, E. coli, Shigella flexneri and Campylobacter jejuni, ${ }^{58} 59$ and butyrate can downregulate expression of genes involved in Salmonella invasion at low doses. ${ }^{60}$ In in vitro fermentations, low iron concentrations decreased numbers of SCFA-producers, including Roseburia species/E. rectale, Clostridium Cluster IV members and Bacteroides species, and decreased butyrate and propionate concentrations in the effluent. ${ }^{61}$ In the present study, we found no effect of iron-containing MNPs on faecal SCFA concentrations. This difference may be due to the fact that $95-99 \%$ of SCFAs produced by bacterial fermentation are absorbed in the colon, so concentrations measured in faeces likely do not reflect levels in the colonic lumen. ${ }^{62}$ In Swedish infants, faecal butyrate and propionate concentrations steadily increased over the first 2 years of life, while faecal acetate increased rapidly in the first few weeks after birth and then plateaued. ${ }^{63}$ A similar pattern from 6 months to 10 months of age was visible in the Kenyan infants in this study.

Data on the effect of iron interventions on diarrhoeal incidence in children disagree. In a systematic review of controlled trials of oral iron supplementation or fortification, provision of iron was associated with a $11 \%$ higher risk of developing diarrhoea $(p=0.04){ }^{7}$ Four food fortification studies reported diarrhoeal outcomes, three provided iron-fortified infant formula, ${ }^{64-66}$ and one provided an iron-fortified infant food. ${ }^{67}$ Since that review, two small iron fortification trials done in school-age children have not reported an increased risk for diarrhoea. $^{68} 69$ However, a controlled trial in Swedish and Honduran infants providing iron supplements from 6 months to 9 months, among infants with $\mathrm{Hb} \geq 110 \mathrm{~g} / \mathrm{L}$, iron treatment increased risk for diarrhoea. ${ }^{70}$ Recent controlled iron supplementation trials $(12.5-15 \mathrm{mg} \mathrm{Fe} /$ day $)$ in $\mathrm{Peru}^{71}$ and Bangladesh $^{72}$ reported a significant increase in diarrhoea. Two large trials of iron and folic acid supplementation in infants and children (subjects aged 12-35 months received $12.5 \mathrm{mg} \mathrm{Fe} /$ day, younger children received half the dose) in $\mathrm{Nepal}^{73}$ and Tanzania $^{3}$ reported no difference in diarrhoea incidence. However, in a controlled study in Ghanaian children that used the same $+12.5 \mathrm{mgFeMNP}$ as in our study, there were significantly more hospital admissions in the iron group (RR (95\% CI) 1.23 (1.02 to 1.49$)$ ), and based on data from the outpatient register, $83 \%$ of the additional cases in the iron group were due to diarrhoea, but this was not significant (RR (95\% CI) 1.12 (0.86 to 1.46$)) .{ }^{5}$ A recent study in Pakistan found an increase in diarrhoea in infants receiving $+12.5 \mathrm{mgFeMNP}$, with or without zinc, compared to untreated controls (without zinc: OR $(95 \%$ CI) 1.15 (1.00 to 1.3$)$; with zinc: 1.31 (1.13 to 1.51$)) .{ }^{6}$ In the present study, there was a trend towards a higher rate of diarrhoea requiring treatment in the infants receiving the $+12.5 \mathrm{mgFeMNP}$. Therefore, the available data suggest oral iron supplements, and +FeMNPs may modestly increase in risk for diarrhoea in infants. Our findings of a shift in the gut microbiome towards a potentially more pathogenic profile along with the increase in intestinal inflammation could provide a potential mechanism for this adverse effect.

This is the first controlled intervention trial to examine the effect of +FeMNPs on the African infant gut microbiome, and our findings need confirmation in other settings and populations. IDA and diarrhoea are major causes of morbidity and mortality in infants in developing countries. In-home fortification with + FeMNPs has repeatedly been shown to reduce IDA rates in infants and children, but whether they increase risk for diarrhoeal disease remains uncertain. The limited available clinical evidence and our findings of their effects on the gut microbiome and inflammation, suggest +FeMNPs may not be entirely safe in settings with high burdens of infectious diseases. Currently, WHO recommends fortification using MNPs containing iron, vitamin A, and zinc for children aged 6-23 months irrespective of their iron status in settings with an anaemia prevalence of $>20 \%$, and in conjunction with measures to treat malaria, promote improved sanitation, and improve overall management of diarrhoea. ${ }^{74}$ At the same time, WHO recommends iron supplementation only be targeted to infants with IDA, while providing adequate protection from malaria. ${ }^{4}$ Our findings, together with others, ${ }^{5} 6$ suggest that, until safer formulations are available, +FeMNPs should be targeted to infants with IDA, while providing adequate protection from malaria and diarrhoea.

Acknowledgements We thank the families who participated in these studies, the project staff for their hard work and dedication; Viola Cassina, Simone Heeb, Alfonso Die, and Erwin Wiegerinck for their support in the lab; and Professor Herbert Haechler from the National Centre for Enteropathogenic Bacteria and Listeria (NENT) for generously proving the DNA of the standard strains for the pathogenic E. coli qPCRs. 
Contributors TJ: study design, study conduct, data collection, data analysis and interpretation, writing of the first draft. GAMK and DM: data analysis and interpretation, writing of the first draft. CC: study design, data interpretation. PH, JN, AM and JK: study conduct and data collection. AD, DWS and CL: data interpretation. JB, HMT and HT: data analysis and interpretation. MBZ: study design, data analysis and interpretation, writing of the first draft. All authors edited the report, approved the final version and declare no conflict of interest. The content is solely the responsibility of the authors and does not necessarily represent the official views of the Eunice Kennedy Shriver National Institute of Child Health \& Human Development or the National Institutes of Health.

Funding This project was supported by the Eunice Kennedy Shriver National Institute Of Child Health \& Human Development under grant number U01HD064921, and realised in collaboration with the INSTAPA Project, funded by the European Union's Seventh Framework Programme (Fp7/2007-2013) under grant agreement number 211484 .

\section{Competing interests None.}

Ethics approval Ethics and research committees of the Kenyatta National Hospital/ University of Nairobi and the ETH Zurich.

Provenance and peer review Not commissioned; externally peer reviewed.

\section{REFERENCES}

1 Adu-Afarwuah S, Lartey A, Brown KH, et al. Home fortification of complementary foods with micronutrient supplements is well accepted and has positive effects on infant iron status in Ghana. Am J Clin Nutr 2008;87:929-38.

2 UNICEF-CDC. Global assessment of home fortification interventions, 2011. Geneva: Home Fortification Technical Advisory Group, 2013.

3 Sazawal S, Black RE, Ramsan M, et al. Effects of routine prophylactic supplementation with iron and folic acid on admission to hospital and mortality in preschool children in a high malaria transmission setting: community-based, randomised, placebo-controlled trial. Lancet 2006;367:133-43.

4 Fontaine 0 . Conclusions and recommendations of the WHO Consultation on prevention and control of iron deficiency in infants and young children in malaria-endemic areas. Food Nutr Bull 2007;28(4 Suppl):S621-7.

5 Zlotkin $\mathrm{S}$, Newton S, Aimone AM, et al. Effect of iron fortification on malaria incidence in infants and young children in Ghana: a randomized trial. JAMA 2013:310:938-47.

6 Soofi S, Cousens S, Iqbal SP, et al. Effect of provision of daily zinc and iron with several micronutrients on growth and morbidity among young children in Pakistan: a cluster-randomised trial. Lancet 2013;382:29-40.

7 Gera T, Sachdev HP. Effect of iron supplementation on incidence of infectious illness in children: systematic review. BMJ 2002;325:1142.

8 WHO. World health statistics 2013. 2013.

9 Zimmermann MB, Hurrell RF. Nutritional iron deficiency. Lancet 2007;370:511-20.

10 Tondeur MC, Schauer CS, Christofides AL, et al. Determination of iron absorption from intrinsically labeled microencapsulated ferrous fumarate (sprinkles) in infants with different iron and hematologic status by using a dual-stable-isotope method. Am J Clin Nutr 2004:80:1436-44.

11 Nemeth E, Tuttle MS, Powelson J, et al. Hepcidin regulates cellular iron efflux by binding to ferroportin and inducing its internalization. Science 2004;306:2090-3.

12 Cassat JE, Skaar EP. Iron in infection and immunity. Cell Host Microbe 2013;13:509-19.

13 Raffatellu M, George MD, Akiyama Y, et al. Lipocalin-2 resistance confers an advantage to Salmonella enterica serotype Typhimurium for growth and survival in the inflamed intestine. Cell Host Microbe 2009:5:476-86.

14 Andrews SC, Robinson AK, Rodriguez-Quinones F. Bacterial iron homeostasis. FEMS Microbiol Rev 2003:27:215-37.

15 Naikare H, Palyada K, Panciera R, et al. Major role for FeoB in Campylobacter jejuni ferrous iron acquisition, gut colonization, and intracellular survival. Infect Immun 2006;74:5433-44.

16 Anderson RC, Cookson AL, McNabb WC, et al. Lactobacillus plantarum DSM 2648 is a potential probiotic that enhances intestinal barrier function. FEMS Microbiol Lett 2010;309:184-92.

17 Weinberg ED. The Lactobacillus anomaly: total iron abstinence. Perspect Biol Med 1997:40:578-83

18 Penders J, Thiijs C, Vink C, et al. Factors influencing the composition of the intestinal microbiota in early infancy. Pediatrics 2006;118:511-21.

19 Yen CC, Shen CJ, Hsu WH, et al. Lactoferrin: an iron-binding antimicrobial protein against Escherichia coli infection. Biometals 2011;24:585-94.

20 Vael C, Desager $\mathrm{K}$. The importance of the development of the intestinal microbiota in infancy. Curr Opin Pediatr 2009;21:794-800.

21 Yatsunenko. Human gut microbiome viewed across age and geography. Nature, 2012

22 Zimmermann MB, Chassard C, Rohner $\mathrm{F}$, et al. The effects of iron fortification on the gut microbiota in African children: a randomized controlled trial in Cote d'Ivoire. Am J Clin Nutr 2010:92:1406-15.
23 Balmer SE, Scott PH, Wharton BA. Diet and faecal flora in the newborn: lactoferrin. Arch Dis Child 1989;64:1685-90.

24 Mevissen-Verhage EA, Marcelis JH, Harmsen-Van Amerongen WC, et al. Effect of iron on neonatal gut flora during the first three months of life. Eur J Clin Microbiol 1985;4:273-8.

25 Krebs NF, Sherlock LG, Westcott J, et al. Effects of different complementary feeding regimens on iron status and enteric microbiota in breastfed infants. J Pediatr 2013:163:416-23.

26 Okiro EA, Hay SI, Gikandi PW, et al. The decline in paediatric malaria admissions on the coast of Kenya. Malar I 2007;6:151.

27 Meilgaard MC, Carr BT, Civille GV. Sensory evaluation techniques. 4th edn. Boca Raton, FL: Taylor Francis Group 2007.

28 Makower RU. Extraction and determination of phytic acid in beans (Phaseolus vulgaris). Albany, California: U.S. Department of Agriculture, 1970.

29 Vanveldhoven PP, Mannaerts GP. Inorgaic and organic phosphate measurements in the nanomolar range. Anal Biochem 1987;161:45-8.

30 WHO. Iron deficiency and anemia: assessment prevention and control. A guide for programme managers. Geneva, Switzerland: World Heath Organization, 2001.

31 Cook JD, Flowers $\mathrm{CH}$, Skikne BS. The quantitative assessment of body iron. Blood 2003;101:3359-64.

32 WHO. Anthro for personal computers. Version 2. Software for assessing growth and development of the world's children. Geneva: World Health Organization, 2007. (http://www.who.int/childgrowth/software/en), 2007.

33 Petry N, Egli I, Chassard C, et al. Inulin modifies the bifidobacteria population, fecal lactate concentration, and fecal $\mathrm{pH}$ but does not influence iron absorption in women with low iron status. Am I Clin Nutr 2012;96:325-31.

34 Dostal A, Baumgartner J, Riesen $\mathrm{N}$, et al. Effects of iron supplementation on dominant bacterial groups in the gut, faecal SCFA and gut inflammation: a randomised, placebo-controlled intervention trial in South African children. Br J Nutr 2014:1-10

35 Bullen J, Griffiths E, Rogers $\mathrm{H}$, et al. Sepsis: the critical role of iron. Microbes Infect 2000:2:409-15.

36 Boyer $\mathrm{E}$, Bergevin I, Malo $\mathrm{D}$, et al. Acquisition of $\mathrm{Mn}(\mathrm{II})$ in addition to $\mathrm{Fe}(\mathrm{II})$ is required for full virulence of Salmonella enterica serovar Typhimurium. Infect Immun 2002:70:6032-42.

37 Tsolis RM, Baumler AJ, Heffron F, et al. Contribution of TonB- and Feo-mediated iron uptake to growth of Salmonella typhimurium in the mouse. Infect Immun 1996;64:4549-56.

38 Pandey A, Bringel F, Meyer J-M. Iron requirement and search for siderophores in lactic acid bacteria. Appl Microbiol Biotechnol 1994;40:735-9.

39 Bezkorovainy A, Solberg L. Ferrous iron uptake by Bifidobacterium breve. Biol Trace Elem Res 1989:20:251-67.

40 Bezkorovainy A, Kot E, Miller-Catchpole R, et al. Iron Metabolism in Bifidobacteria. Int Dairy Journal 1996;6:905-19.

41 Coconnier MH, Lievin V, Bernet-Camard MF, et al. Antibacterial effect of the adhering human Lactobacillus acidophilus strain LB. Antimicrob Agents Chemother 1997;41:1046-52.

42 Lievin V, Peiffer I, Hudault $\mathrm{S}$, et al. Bifidobacterium strains from resident infant human gastrointestinal microflora exert antimicrobial activity. Gut 2000:47:646-52.

43 Stecher B, Chaffron S, Kappeli R, et al. Like will to like: abundances of closely related species can predict susceptibility to intestinal colonization by pathogenic and commensal bacteria. PLoS Pathog 2010;6:e1000711.

44 Gisbert JP, McNicholl AG. Questions and answers on the role of faecal calprotectin as a biological marker in inflammatory bowel disease. Dig Liver Dis 2009:41:56-66

45 Berni Canani R, Rapacciuolo L, Romano MT, et al. Diagnostic value of faecal calprotectin in paediatric gastroenterology clinical practice. Dig Liver Dis 2004:36:467-70.

46 Konikoff MR, Denson LA. Role of fecal calprotectin as a biomarker of intestinal inflammation in inflammatory bowel disease. Inflamm Bowel Dis 2006:12:524-34

47 Hestvik E, Tumwine JK, Tylleskar T, et al. Faecal calprotectin concentrations in apparently healthy children aged $0-12$ years in urban Kampala, Uganda: a community-based survey. BMC Pediatr 2011;11:9

48 Maynard CL, Elson CO, Hatton RD, et al. Reciprocal interactions of the intestinal microbiota and immune system. Nature 2012;489:231-41.

49 Carrier JC, Aghdassi E, Jeejeebhoy $\mathrm{K}$, et al. Exacerbation of dextran sulfate sodium-induced colitis by dietary iron supplementation: role of NF-kappaB. Int $J$ Colorectal Dis 2006:21:381-7.

50 Winter SE, Lopez CA, Baumler AJ. The dynamics of gut-associated microbial communities during inflammation. EMBO Rep 2013;14:319-27.

51 Simpson RJ, Sidhar S, Peters TJ. Application of selective extraction to the study of iron species present in diet and rat gastrointestinal tract contents. Br J Nutr 1992:67:437-44.

52 Koster W. ABC transporter-mediated uptake of iron, siderophores, heme and vitamin B12. Res Microbiol 2001;152:291-301. 
53 De Filippo C, Cavalieri D, Di Paola M, et al. Impact of diet in shaping gut microbiota revealed by a comparative study in children from Europe and rural Africa. Proc Natl Acad Sci USA 2010;107:14691-6.

54 Backhed F, Ley RE, Sonnenburg JL, et al. Host-bacterial mutualism in the human intestine. Science 2005;307:1915-20.

55 Qin J, Li R, Raes J, et al. A human gut microbial gene catalogue established by metagenomic sequencing. Nature 2010;464:59-65.

56 Fallani M, Amarri S, Uusijarvi A, et al. Determinants of the human infant intestinal microbiota after the introduction of first complementary foods in infant samples from five European centres. Microbiology 2011;157(Pt 5):1385-92.

57 Dostal A, Chassard C, Hilty FM, et al. Iron depletion and repletion with ferrous sulfate or electrolytic iron modifies the composition and metabolic activity of the gut microbiota in rats. J Nutr 2012;142:271-7.

58 Topping DL, Clifton PM. Short-chain fatty acids and human colonic function: roles of resistant starch and nonstarch polysaccharides. Physiol Rev 2001;81:1031-64.

59 Ricke SC. Perspectives on the use of organic acids and short chain fatty acids as antimicrobials. Poult Sci 2003;82:632-9.

60 Van Immerseel F, Russell JB, Flythe MD, et al. The use of organic acids to combat Salmonella in poultry: a mechanistic explanantion of the efficacy. Avian Pathol 2006;35:182-8.

61 Dostal A, Fehlbaum S, Chassard C, et al. Low iron availability in continuous in vitro colonic fermentations induces strong dysbiosis of the child gut microbial consortium and a decrease in main metabolites. FEMS Microbiol Ecol 2013;83:161-75.

62 Scheppach W. Effects of short chain fatty acids on gut morphology and function. Gut 1994;35(1 Suppl):S35-8.

63 Midtvedt AC, Midtvedt T. Production of short chain fatty acids by the intestinal microflora during the first 2 years of human life. J Pediatr Gastroenterol Nutr 1992;15:395-403.

64 Power $\mathrm{HM}$, Heese HD, Beatty DW, et al. Iron fortification of infant milk formula: the effect on iron status and immune function. Annals Trop Paediatr 1991;11:57-66.
65 Brunser O, Espinoza J, Araya M, et al. Chronic iron intake and diarrhoeal disease in infants. A field study in a less-developed country. Eur J Clin Nutr 1993;47:317-26.

66 Singhal A, Morley R, Abbott $R$, et al. Clinical safety of iron-fortified formulas. Pediatrics 2000;105:E38.

67 Javaid N, Haschke F, Pietschnig B, et al. Interactions between infections, malnutrition and iron nutritional status in Pakistani infants. A longitudinal study. Acta Paediatr Scand Suppl 1991;374:141-50.

68 Moretti D, Zimmermann MB, Muthayya S, et al. Extruded rice fortified with micronized ground ferric pyrophosphate reduces iron deficiency in Indian schoolchildren: a double-blind randomized controlled trial. Am J Clin Nutr 2006;84:822-9.

69 Manger MS, McKenzie JE, Winichagoon P, et al. A micronutrient-fortified seasoning powder reduces morbidity and improves short-term cognitive function, but has no effect on anthropometric measures in primary school children in northeast Thailand: a randomized controlled trial. Am J Clin Nutr 2008;87:1715-22.

70 Dewey KG, Domellof $\mathrm{M}$, Cohen RJ, et al. Iron supplementation affects growth and morbidity of breast-fed infants: results of a randomized trial in Sweden and Honduras. J Nutr 2002;132:3249-55.

71 Richard SA, Zavaleta N, Caulfield LE, et al. Zinc and iron supplementation and malaria, diarrhea, and respiratory infections in children in the Peruvian Amazon. Am J Trop Med Hyg 2006;75:126-32.

72 Chang S, El Arifeen S, Bari S, et al. Supplementing iron and zinc: double blind, randomized evaluation of separate or combined delivery. Eur I Clin Nutr 2010;64:153-60

73 Tielsch JM, Khatry SK, Stoltzfus RJ, et al. Effect of routine prophylactic supplementation with iron and folic acid on preschool child mortality in southern Nepal: community-based, cluster-randomised, placebo-controlled trial. Lancet 2006:367:144-52.

74 WHO. Guideline: use of multiple micronutrient powders for home fortification of foods consumed by infants and children 6-23 months of age. Geneva, World Health Organization, 2011. 